\title{
Greening of the Earth and its drivers
}

\author{
Zaichun Zhu ${ }^{1,2}$, Shilong Piao ${ }^{1,2 \star}{ }^{\text {, Ranga B. Myneni }}{ }^{3}$, Mengtian Huang ${ }^{2}$, Zhenzhong Zeng ${ }^{2}$, \\ Josep G. Canadell ${ }^{4}$, Philippe Ciais ${ }^{2,5}$, Stephen Sitch ${ }^{6}$, Pierre Friedlingstein ${ }^{7}$, Almut Arneth ${ }^{8}$, \\ Chunxiang Cao ${ }^{9}$, Lei Cheng ${ }^{10}$, Etsushi Kato ${ }^{11}$, Charles Koven ${ }^{12}$, Yue Li' ${ }^{2}$, Xu Lian ${ }^{2}$, Yongwen Liu ${ }^{2}$, \\ Ronggao Liu' ${ }^{13}$, Jiafu Mao ${ }^{14}$, Yaozhong Pan ${ }^{15}$, Shushi Peng ${ }^{2}$, Josep Peñuelas ${ }^{16,17}$, Benjamin Poulter ${ }^{18}$, \\ Thomas A. M. Pugh ${ }^{8,19}$, Benjamin D. Stocker ${ }^{20,21}$, Nicolas Viovy ${ }^{5}$, Xuhui Wang ${ }^{2}$, Yingping Wang ${ }^{22}$, \\ Zhiqiang Xiao ${ }^{23}$, Hui Yang ${ }^{2}$, Sönke Zaehle ${ }^{24}$ and Ning Zeng ${ }^{25}$
}

\begin{abstract}
Global environmental change is rapidly altering the dynamics of terrestrial vegetation, with consequences for the functioning of the Earth system and provision of ecosystem services ${ }^{1,2}$. Yet how global vegetation is responding to the changing environment is not well established. Here we use three long-term satellite leaf area index (LAI) records and ten global ecosystem models to investigate four key drivers of LAI trends during 1982-2009. We show a persistent and widespread increase of growing season integrated LAl (greening) over $25 \%$ to $50 \%$ of the global vegetated area, whereas less than $4 \%$ of the globe shows decreasing LAl (browning). Factorial simulations with multiple global ecosystem models suggest that $\mathrm{CO}_{2}$ fertilization effects explain $70 \%$ of the observed greening trend, followed by nitrogen deposition ( $9 \%)$, climate change ( $8 \%$ ) and land cover change (LCC) $(4 \%)$. $\mathrm{CO}_{2}$ fertilization effects explain most of the greening trends in the tropics, whereas climate change resulted in greening of the high latitudes and the Tibetan Plateau. LCC contributed most to the regional greening observed in southeast China and the eastern United States. The regional effects of unexplained factors suggest that the next generation of ecosystem models will need to explore the impacts of forest demography, differences in regional management intensities for cropland and pastures, and other emerging productivity constraints such as phosphorus availability.
\end{abstract}

Changes in vegetation greenness have been reported at regional and continental scales on the basis of forest inventory and satellite measurements ${ }^{3-8}$. Long-term changes in vegetation greenness are driven by multiple interacting biogeochemical drivers and land-use effects $^{9}$. Biogeochemical drivers include the fertilization effects of elevated atmospheric $\mathrm{CO}_{2}$ concentration $\left(\mathrm{eCO}_{2}\right)$, regional climate change (temperature, precipitation and radiation), and varying rates of nitrogen deposition. Land-use-related drivers involve changes in land cover and in land management intensity, including fertilization, irrigation, forestry and grazing ${ }^{10}$. None of these driving factors can be considered in isolation, given their strong interactions with one another. Previously, a few studies had investigated the drivers of global greenness trends ${ }^{6,7,11}$, with a limited number of models and satellite observations, which prevented an appropriate quantification of uncertainties ${ }^{12}$.

Here, we investigate trends of leaf area index (LAI) and their drivers for the period 1982 to 2009 using three remotely sensed data sets (GIMMS3g, GLASS and GLOMAP) and outputs from ten ecosystem models run at global extent (see Supplementary Information). We use the growing season integrated leaf area index (hereafter, LAI; Methods) as the variable of our study. We first analyse global and regional LAI trends for the study period and differences between the three data sets. Using modelling results, we then quantify the contributions of $\mathrm{CO}_{2}$ fertilization, climatic factors, nitrogen deposition and LCC to the observed trends.

Trends from the three long-term satellite LAI data sets consistently show positive values over a large proportion of the global vegetated area since 1982 (Fig. 1). The global greening trend estimated from the three data sets is $0.068 \pm 0.045 \mathrm{~m}^{2} \mathrm{~m}^{-2} \mathrm{yr}^{-1}$.

\footnotetext{
${ }^{1}$ Key Laboratory of Alpine Ecology and Biodiversity, Institute of Tibetan Plateau Research, CAS Center for Excellence in Tibetan Plateau Earth Science, Chinese Academy of Sciences, Beijing 100085, China. ${ }^{2}$ Sino-French Institute for Earth System Science, College of Urban and Environmental Sciences, Peking University, Beijing 100871, China. ${ }^{3}$ Department of Earth and Environment, Boston University, Boston, Massachusetts 02215, USA. ${ }^{4} \mathrm{Global}$ Carbon Project, CSIRO Oceans and Atmosphere, GPO Box 3023, Canberra, Australian Capital Territory 2601, Australia. ${ }^{5}$ Laboratoire des Sciences du Climat et de I'Environnement (LSCE), CEA CNRS UVSQ, 91191 Gif Sur Yvette, France. ${ }^{6}$ College of Life and Environmental Sciences, University of Exeter, Exeter EX4 4QF, UK. ${ }^{7}$ College of Engineering, Mathematics and Physical Sciences, University of Exeter, Exeter EX4 4QF, UK. ${ }^{8}$ Institute of Meteorology and Climate Research, Atmospheric Environmental Research, Karlsruhe Institute of Technology, 82467 Garmisch-Partenkirchen, Germany. ${ }^{9}$ State Key Laboratory of Remote Sensing Science, Institute of Remote Sensing and Digital Earth, Chinese Academy of Sciences, Beijing 100101, China. ${ }^{10} \mathrm{CSIRO}$ Land and Water, Black Mountain, Canberra, Australian Capital Territory 2601, Australia. ${ }^{11}$ Institute of Applied Energy (IAE), Minato-ku, Tokyo 105-0003, Japan. ${ }^{12}$ Earth Sciences Division, Lawrence Berkeley National Lab, 1 Cyclotron Road, Berkeley, California 94720, USA. ${ }^{13}$ LREIS, Institute of Geographic Sciences and Natural Resources Research, Chinese Academy of Sciences, Beijing 100101, China. ${ }^{14}$ Climate Change Science Institute and Environmental Sciences Division, Oak Ridge National Laboratory, Oak Ridge, Tennessee 37831, USA. ${ }^{15}$ College of Resources Science \& Technology, State Key Laboratory of Earth Processes and Resource Ecology, Beijing Normal University, Beijing 100875, China. ${ }^{16}$ CSIC, Global Ecology Unit CREAF-CEAB-UAB, Cerdanyola del Vallès, 08193 Catalonia, Spain. ${ }^{17}$ CREAF, Cerdanyola del Vallès, 08193 Catalonia, Spain. ${ }^{18}$ Montana State University, Institute on Ecosystems and the Department of Ecology, Bozeman, Montana 59717, USA. ${ }^{19}$ School of Geography, Earth and Environmental Science, University of Birmingham, Birmingham B15 2TT, UK. ${ }^{20}$ Department of Life Sciences, Imperial College London, Silwood Park, Ascot SL5 7PY, UK. ${ }^{21}$ Climate and Environmental Physics, and Oeschger Centre for Climate Change Research, University of Bern, 3012 Bern, Switzerland. ${ }^{22}$ CSIRO Oceans and Atmosphere, PMB \#1, Aspendale, Victoria 3195, Australia. ${ }^{23}$ State Key Laboratory of Remote Sensing Science, School of Geography, Beijing Normal University, Beijing 100875, China. ${ }^{24}$ Max-Planck-Institut für Biogeochemie, PO Box 600164, Hans-Knöll-Str. 10, 07745 Jena, Germany. ${ }^{25}$ Department of Atmospheric and Oceanic Science, University of Maryland, College Park, Maryland 20742, USA. *e-mail: slpiao@pku.edu.cn
} 
a
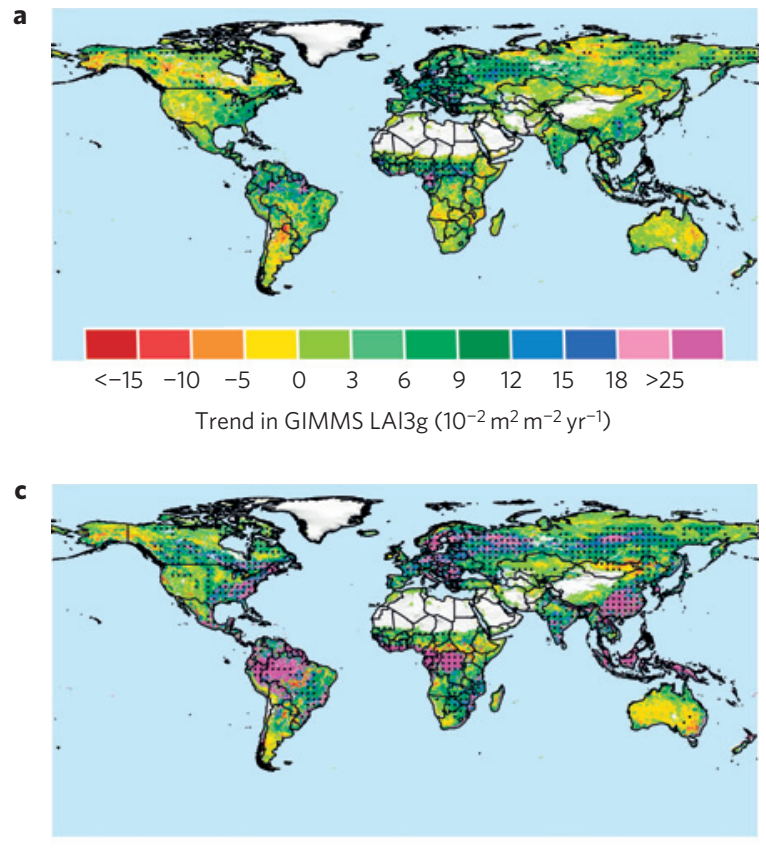

Trend in GLASS LAI $\left(10^{-2} \mathrm{~m}^{2} \mathrm{~m}^{-2} \mathrm{yr}^{-1}\right)$ b

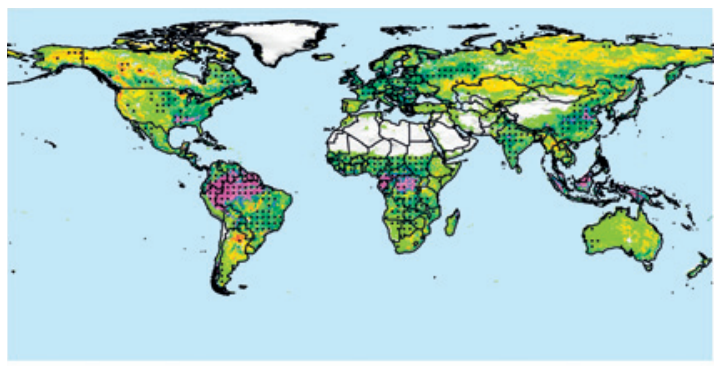

Trend in GLOBMAP LAI $\left(10^{-2} \mathrm{~m}^{2} \mathrm{~m}^{-2} \mathrm{yr}^{-1}\right)$

d

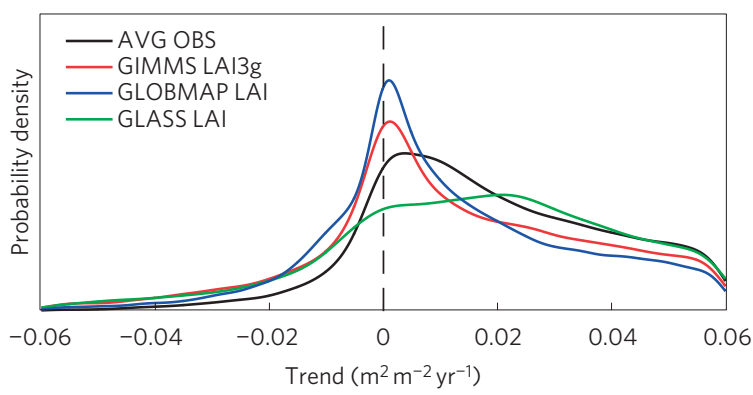

Figure 1 | Trend in observed growing season integrated LAI. a-c, Spatial pattern of trends in growing season integrated LAI derived from three remote sensing data sets. a, GIMMS LAI3g. b, GLOBMAP LAI. c, GLASS LAI. All data sets cover the period 1982 to 2009 . Regions labelled by black dots indicate trends that are statistically significant (Mann-Kendall test; $p<0.05$ ). d, Probability density function of LAI trends for GIMMS LAI3g, GLASS LAI, GLOBMAP LAI and the average of the three remote sensing data sets (AVG OBS).

The GIMMS LAI3g data set, which includes recent data up to 2014, shows a continuation of the trend from the 1982 to 2009 period (Fig. 1 and Supplementary Fig. 3). The regions with the largest greening trends, consistent across the three data sets, are in southeast North America, the northern Amazon, Europe, Central Africa and Southeast Asia. The GLASS LAI data shows the most extensive statistically significant greening (Mann-Kendall test, $p<0.05$ ) over $50 \%$ of vegetated lands, followed by GLOBMAP LAI (43\%) and GIMMS LAI3g (25\%). All three LAI data sets also consistently show a decreasing LAI trend (browning) over less than $4 \%$ of global vegetated land-these are observed in northwest North America and central South America. Analyses of the changes in observed maximum LAI also show similar widespread greening trends (Supplementary Section 8).

We compare satellite-based LAI anomalies with LAI anomalies simulated by ten global ecosystem models driven by $\mathrm{eCO}_{2}$ (+46 ppm over the study period), climate, nitrogen deposition and LCC (Supplementary Section 7). Multi-Model Ensemble Mean (MMEM) LAI anomalies, with all these drivers considered, generally agree with averaged satellite observations at the global scale $(r=0.85, p<0.01$; Fig. 2a). The trend in MMEM LAI anomalies $\left(0.062 \mathrm{~m}^{2} \mathrm{~m}^{-2} \mathrm{yr}^{-1}\right)$ is within the range of estimates from the three satellite data sets. The model simulations suggest that increasing gross primary productivity, although partly neutralized by increasing autotrophic respiration, and decreasing carbon loss due to fires are responsible for the increasing LAI during 1982 to 2009 (Supplementary Section 9). The spatial pattern of LAI trends also matches well between satellite data and MMEM simulations (Fig. 3a,b). Consistent greening trends between models and observations are seen in Fig. 3 across the southeast United States, the Amazon Basin, Europe, central Africa, Southeast Asia and Australia. However, satellite LAI and MMEM results show different magnitudes (or signs) of trends in the southwestern United States, southern South American countries, and Mongolia, indicating that models may be over-sensitive to trends in precipitation (Supplementary Section 10).
We used an optimal fingerprint detection method ${ }^{13}$ to assess the ability of the models to simulate response patterns of LAI to $\mathrm{eCO}_{2}$, climate change, nitrogen deposition and LCC. We regressed the observed two-year mean global average LAI time series against the MMEM-simulated LAI reflecting the effects of single drivers, based on factorial runs where only one driver is varied at the time. A residual consistency test ${ }^{13}$ suggests no inconsistency between the regression residuals and the modelsimulated internal variability in the absence of forcing (Methods), indicating that the fingerprint detection method is suitable for detection and attribution at the global scale (Fig. 2b). The 95\% confidence intervals of the scaling factors of $\mathrm{CO}_{2}$ fertilization (best estimates of scaling factor $\beta=1.03,95 \%$ confidence interval $[0.84,1.23])$ and climate change $(\beta=1.06,[0.55,1.64])$ are not only above zero but also span unity, which means that the modelled signals from these two drivers are successfully detected and suitable for attribution (Fig. 2b). The fingerprints of nitrogen deposition and LCC effects on the trend of LAI remain confounded with internal variability and cannot be clearly detected (not shown).

Globally, the model factorial simulations suggest that $\mathrm{CO}_{2}$ fertilization explains the largest contribution to the satellite-observed LAI trend $\left(70.1 \pm 29.4 \%, 0.048 \pm 0.020 \mathrm{~m}^{2} \mathrm{~m}^{-2} \mathrm{yr}^{-1}\right)$, followed by nitrogen deposition $\left(8.8 \pm 11.8 \%, 0.006 \pm 0.008 \mathrm{~m}^{2} \mathrm{~m}^{-2} \mathrm{yr}^{-1}\right)$, climate change $\left(8.1 \pm 20.6 \%, 0.006 \pm 0.014 \mathrm{~m}^{2} \mathrm{~m}^{-2} \mathrm{yr}^{-1}\right)$ and LCC $\left(3.7 \pm 14.7 \%, 0.003 \pm 0.010 \mathrm{~m}^{2} \mathrm{~m}^{-2} \mathrm{yr}^{-1}\right)$ (Fig. 2c). The contributions of $\mathrm{CO}_{2}$ fertilization and climate change are reliable according to the optimal fingerprint analysis, whereas the effects of LCC and nitrogen deposition should be interpreted with caution. Our estimation of $\mathrm{CO}_{2}$ fertilization effects on vegetation growth is more prominent than $\operatorname{Los}^{6}$, probably owing to the different attribution approaches. When using only those ecosystem models (five out of ten) that incorporate nitrogen limitations and nitrogen deposition effects (Supplementary Table 1), the fraction of the LAI trend that is unambiguously attributed to $\mathrm{CO}_{2}$ fertilization is 


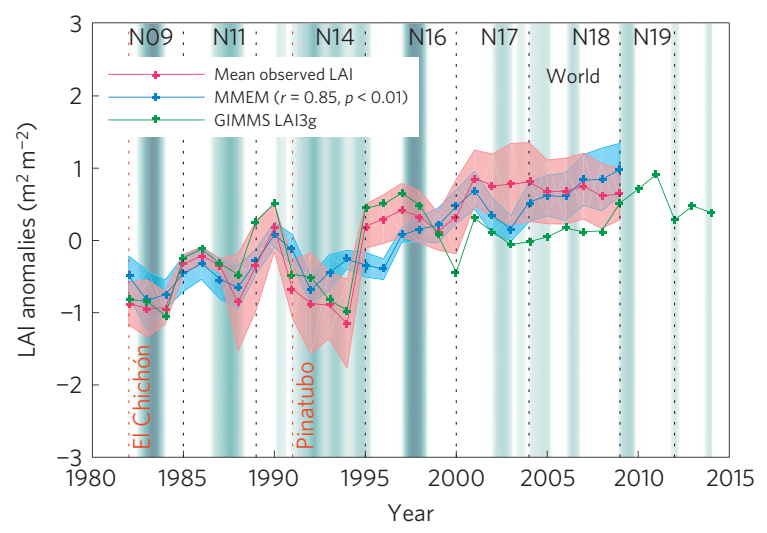

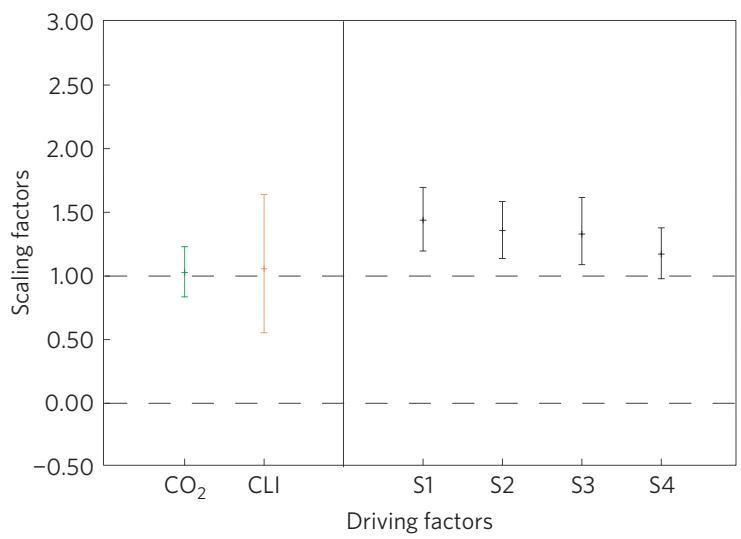

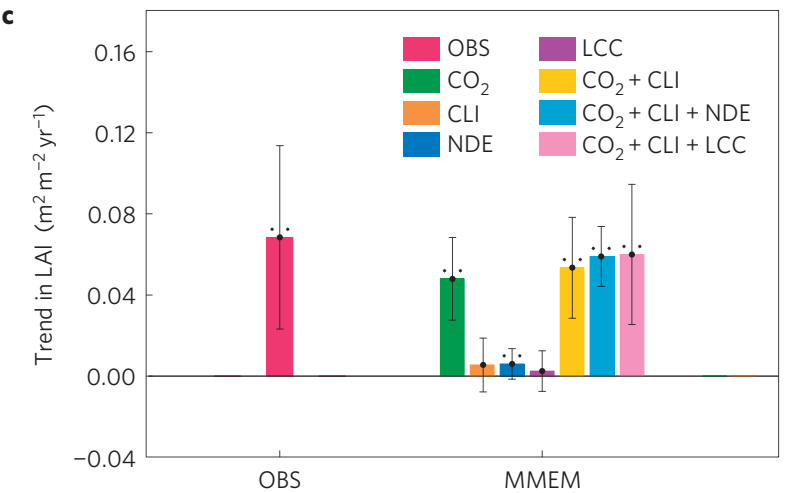

Figure 2 | Attribution of trend in growing season integrated LAI. a, Interannual changes in anomalies of growing season integrated LAI estimated by multi-model ensemble mean (MMEM) with all drivers considered (blue line) and the average of the three remote sensing data (red line) for the period 1982-2009, and the interannual changes in anomalies of LAI of GIMMS LAI3g (green line) for the period 1982-2014. The shaded area shows the intensity

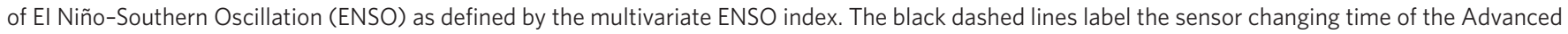
Very High Resolution Radiometer (AVHRR) satellite series. Two volcanic eruptions (El Chichón eruption and Pinatubo eruption) are indicated with red dashed lines. $\mathbf{b}$, Best estimates of the scaling factors of $\mathrm{CO}_{2}$ fertilization effects $\left(\mathrm{CO}_{2}\right)$, climate change effects (CLI) and simulated LAI under the four scenarios (see Methods for more details) and their 5-95\% uncertainty range from optimal fingerprint analyses of global LAI for 1982-2009. c, Trend in global-averaged LAI derived from satellite observation (OBS) and modelled trends driven by rising $\mathrm{CO}_{2}\left(\mathrm{CO}_{2}\right)$, climate change (CLI), nitrogen deposition (NDE) and land cover change (LCC) using the Mann-Kendall test. Error bars show the standard deviation of trends derived from satellite data and model simulations. Two asterisks indicate that the trend is statistically significant $(p<0.05)$.

slightly smaller $\left(66.2 \pm 13.2 \%, 0.045 \pm 0.009 \mathrm{~m}^{2} \mathrm{~m}^{-2} \mathrm{yr}^{-1}\right)$ than when using models that ignore nitrogen processes $(75.0 \pm 42.6 \%$, $\left.0.051 \pm 0.029 \mathrm{~m}^{2} \mathrm{~m}^{-2} \mathrm{yr}^{-1}\right)$. This suggests that, although incorporating nitrogen in ecosystem models does not significantly ( $t$-test, $p<0.05)$ change the contribution of the $\mathrm{CO}_{2}$ fertilization effects to the global trend of LAI, it reduces the spread of model simulations $(F$-test, $p<0.05)$.

Vegetation leaf area changes result from interacting factors, but factorial simulations help to attribute a dominant factor for the observed changes. Our analyses show that the $\mathrm{CO}_{2}$ fertilization effects have a rather spatially uniform effect on the positive LAI trends. The modelled relative increases in global mean LAI due to $\mathrm{CO}_{2}$ fertilization alone is about 4.7-9.5\% (or 10.2$20.7 \%$ per $100 \mathrm{ppm}$ ) during 1982 to 2009 , which is comparable to measurements from the Free-Air $\mathrm{CO}_{2}$ Enrichment (FACE) experiments $(0.3-11.1 \% \text {, or } 0.6-24.1 \% \text { per } 100 \mathrm{ppm})^{14}$. However, no FACE experiment covered tropical forests, where models suggest that $\mathrm{eCO}_{2}$ is the dominant factor of the recent LAI trend (Fig. 3c,d). The spatial pattern is consistent with previous analyses ${ }^{15}$ that posited large absolute LAI increases due to $\mathrm{eCO}_{2}$ in the tropics, in the absence of temperature, water and nitrogen limitations ${ }^{16}$, and large relative LAI increases due to $\mathrm{eCO}_{2}$ in arid regions, where $\mathrm{eCO}_{2}$ is expected to increase the water use efficiency of plants (Supplementary Fig. 12) ${ }^{17}$. A simple theoretical model ${ }^{17,18}$ was used to diagnose the response of leaf level carbon assimilation to the observed $46 \mathrm{ppm}$ increase of $\mathrm{CO}_{2}$ over the study period, including the effect of vapour pressure deficit trends and stomatal closure. This model gave a similar relative response of carbon assimilation to $\mathrm{eCO}_{2}$ as the ecosystem models did for LAI (Supplementary Section 12).

Climate change explains about $8.1 \pm 20.1 \%$ of the observed positive LAI trend but, unlike $\mathrm{eCO}_{2}$ effects, climatic effects are negative in some regions. Although detected by the optimal fingerprint model, the effects of climate change are not consistent between models, and may even be opposite in individual model simulations. Overall, climate change has dominant contributions to the greening trend over $28.4 \%$ of the global vegetated area (Fig. 3c,d). Positive effects of climate change in the northern high latitudes and the Tibetan Plateau are attributed to rising temperature, which enhances photosynthesis and lengthens the growing season ${ }^{5}$, whereas the greening of the Sahel and South Africa are primarily driven by increasing precipitation (Supplementary Fig. 13). South America is the only continent where negative climate effects were statistically significant (Supplementary Figs 10 and $11 \mathrm{~b}$ ). This is particularly important owing to the role of the Amazon forests in the global carbon cycle $^{19,20}$. Ecosystem models may tend to overestimate the responses of vegetation growth to precipitation ${ }^{12}$ (Supplementary Section 10), which is one of the reasons why the fate of the Amazon forests continues to be debated ${ }^{10}$. 
a
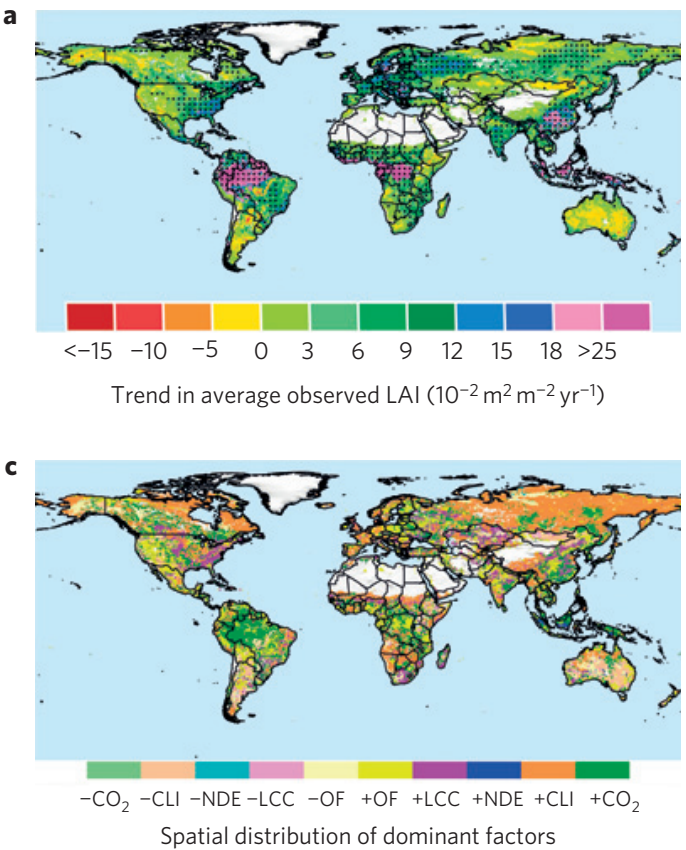

b

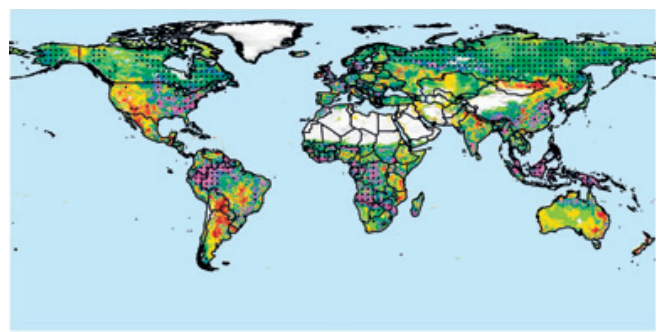

Trend in MMEM LAI $\left(10^{-2} \mathrm{~m}^{2} \mathrm{~m}^{-2} \mathrm{yr}^{-1}\right)$

d

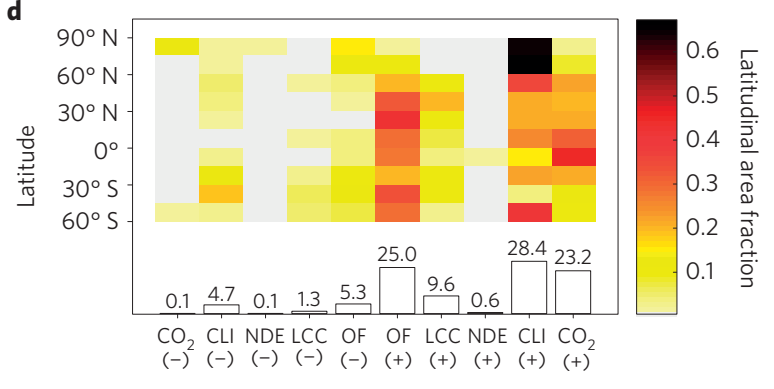

Figure $\mathbf{3}$ | Spatial pattern of dominant drivers of trend in growing season integrated LAl. a,b, Spatial distribution pattern of the trend in growing season integrated LAI for the period 1982-2009. LAI trends were derived from the average of GIMMS, GLOBMAP and GLASS LAI in a and from a multi-model ensemble mean with all drivers considered in $\mathbf{b}$; regions labelled by dots have trends that are statistically significant $(p<0.05)$. The trend is calculated and evaluated using the Mann-Kendall test at the $5 \%$ significance level. c, Dominant driving factors of LAI, defined as the driving factor that contributes the most to the increase (or decrease) in LAl in each vegetated grid cell. The driving factors include rising $\mathrm{CO}_{2}\left(\mathrm{CO}_{2}\right)$, climate change (CLI), nitrogen deposition (NDE), land cover change (LCC) and other factors (OF), the latter being defined by the non-modelled fraction of observed LAI trend (see text). A prefix ' + ' of the driving factors indicates a positive effect on LAI trends, whereas ' - ' indicates a negative effect. d, Fractional area of vegetated land in $15^{\circ}$ latitude bands $\left(90^{\circ} \mathrm{N}-60^{\circ} \mathrm{S}\right)$ attributed to different factors. The fraction of vegetated area (\%) that is dominantly driven by each factor is labelled on top of the bar; + and - have the same meaning as in c.

Considerable evidence points to nitrogen limitation of vegetation growth over many parts of the Earth ${ }^{21}$, with local alleviation by nitrogen deposition in boreal and temperate regions ${ }^{22,23}$. Our analyses suggest that nitrogen deposition explains $8.8 \pm 11.8 \%$ of the LAI trend at the global scale. However, this result is uncertain, because only two models in the ensemble specifically performed factorial simulations with and without nitrogen deposition. A slightly negative trend in nitrogen deposition effect was observed in North America and Europe, where nitrogen deposition rates have stabilized, or even declined, during the past three decades $s^{24,25}$.

LCC is a dominant driver of LAI greening over only $9.6 \%$ of the global vegetated area, mainly in southeast China and southeast United States. Models produce negative LCC effects on LAI trends in tropical and southern temperate regions where deforestation occurred (Supplementary Fig. 11d) ${ }^{26}$. However, the individual effect of LCC is apparently outweighed by other factors in these regions, and thus does not seem to be dominant. Trends of the LCC effect simulated by ecosystem models differ significantly in magnitude, and sometimes also in sign. This could be due to differences in model assumptions relating to whether the productivity of secondary vegetation is smaller or larger than that of the vegetation it replaces.

At the global scale, the observed LAI trend can be largely accounted for by $\mathrm{eCO}_{2}$, climate change, nitrogen deposition and LCC. However, at regional scales, other factors (OF) not considered in models, such as forest management, grazing, changes in cultivation practices and varieties, irrigation and disturbances such as storms and insect attacks, can be a cause of mismatch between observed and simulated LAI trends. The patterns of the effect of other factors were estimated as a residual, by subtracting the simulated trend caused by factors explicitly modelled from the observed local LAI trend. OF contributes the most to the observed LAI trend over 25.0\% (increase) and 5.3\% (decrease) of the vegetated area (Fig. 3d). OF can also encompass non-modelled processes, such as plant diversity within a type of vegetation, hydrological and nutrient liberation during permafrost thawing, phosphorus and potassium limitations, access to ground water by deep roots, and rigid discretization of the simulated vegetation into few plant functional types. Further, uncertainties in existing model parameterization and structure (Supplementary Section 7) and biases from the remote sensing data sets (Supplementary Section 6) can cause a mismatch between simulated and observed LAI trends. Interestingly, positive effects tentatively attributed to OF are mainly found in areas of intensive ecosystem management, such as northeast China, Europe and India ${ }^{27}$. Negative OF effects are mainly found in northern high latitudes, where most models lack a representation of regionally important ecosystems (peatlands, wetlands) as well as of specific disturbances ${ }^{28,29}$.

Understanding the mechanisms behind LAI trends is a first, yet critical, step towards better understanding the influence of human actions on terrestrial vegetation, and towards improving future projections of vegetation dynamics. By making use of three LAI data sets, an ensemble of ten ecosystem models, and a fingerprinting technique, we assessed the consistency of observed greening and browning patterns with the effects of key environmental drivers. The use of a ten-model ensemble increases confidence in the attribution, although model simulations diverge in some aspects, particularly for the impacts of climate change and LCC, which suggests an area for future model improvements. Overall, the described LAI trends represent a significant alteration of the productive capacity of terrestrial vegetation through anthropogenic influences. 


\section{Methods}

Methods and any associated references are available in the online version of the paper.

\section{Received 8 June 2015; accepted 29 March 2016; published online 25 April 2016}

\section{References}

1. Peters, G. P. et al. The challenge to keep global warming below $2^{\circ}$. Nature Clim. Change 3, 4-6 (2013).

2. Ciais, P. et al. in Climate Change 2013: The Physical Science Basis (eds Stocker, T. F. et al.) Ch. 6 (IPCC, Cambridge Univ. Press, 2013).

3. Myneni, R. B., Keeling, C. D., Tucker, C. J., Asrar, G. \& Nemani, R. R. Increased plant growth in the northern high latitudes from 1981 to 1991. Nature 386, 698-702 (1997)

4. Pan, Y. et al. A large and persistent carbon sink in the world's forests. Science 333, 988-993 (2011).

5. Xu, L. et al. Temperature and vegetation seasonality diminishment over northern lands. Nature Clim. Change 3, 581-586 (2013).

6. Los, S. O. Analysis of trends in fused AVHRR and MODIS NDVI data for 1982-2006: indication for a $\mathrm{CO}_{2}$ fertilization effect in global vegetation. Glob. Biogeochem. Cycles 27, 318-330 (2013).

7. Mao, J. F. et al. Global latitudinal-asymmetric vegetation growth trends and their driving mechanisms: 1982-2009. Remote Sens. 5, 1484-1497 (2013).

8. Piao, S. et al. Detection and attribution of vegetation greening trend in China over the last 30 years. Glob. Change Biol. 21, 1601-1609 (2015).

9. Wang, X. H. et al. A two-fold increase of carbon cycle sensitivity to tropical temperature variations. Nature 506, 212-215 (2014).

10. Malhi, Y. et al. Climate change, deforestation, and the fate of the Amazon. Science 319, 169-172 (2008).

11. Ukkola, A. M. et al. Reduced streamflow in water-stressed climates consistent with $\mathrm{CO}_{2}$ effects on vegetation. Nature Clim. Change 6, 75-78 (2015).

12. Piao, S. L. et al. Evaluation of terrestrial carbon cycle models for their response to climate variability and to $\mathrm{CO}_{2}$ trends. Glob. Change Biol. 19, 2117-2132 (2013).

13. Allen, M. R. \& Tett, S. F. B. Checking for model consistency in optimal fingerprinting. Clim. Dynam. 15, 419-434 (1999).

14. Norby, R. J. et al. Forest response to elevated $\mathrm{CO}_{2}$ is conserved across a broad range of productivity. Proc. Natl Acad. Sci. USA 102, 18052-18056 (2005).

15. Schimel, D., Stephens, B. B. \& Fisher, J. B. Effect of increasing $\mathrm{CO}_{2}$ on the terrestrial carbon cycle. Proc. Natl Acad. Sci. USA 112, 436-441 (2015).

16. Galloway, J. N. et al. Nitrogen cycles: past, present, and future. Biogeochemistry 70, 153-226 (2004).

17. Donohue, R. J., Roderick, M. L., McVicar, T. R. \& Farquhar, G. D. Impact of $\mathrm{CO}_{2}$ fertilization on maximum foliage cover across the globe's warm, arid environments. Geophys. Res. Lett. 40, 3031-3035 (2013).

18. Wong, S. C., Cowan, I. R. \& Farquhar, G. D. Stomatal conductance correlates with photosynthetic capacity. Nature 282, 424-426 (1979).

19. Achard, F. et al. Determination of deforestation rates of the world's humid tropical forests. Science 297, 999-1002 (2002).

20. Gibson, L. et al. Primary forests are irreplaceable for sustaining tropical biodiversity. Nature 478, 378-381 (2011).
21. LeBauer, D. S. \& Treseder, K. K. Nitrogen limitation of net primary productivity in terrestrial ecosystems is globally distributed. Ecology 89, 371-379 (2008).

22. Magnani, F. et al. The human footprint in the carbon cycle of temperate and boreal forests. Nature 447, 848-850 (2007).

23. Canadell, J. G. \& Schulze, E. D. Global potential of biospheric carbon management for climate mitigation. Nature Commun. 5, 5282 (2014).

24. Goulding, K. W. T. et al. Nitrogen deposition and its contribution to nitrogen cycling and associated soil processes. New Phytol. 139, 49-58 (1998).

25. Holland, E. A., Braswell, B. H., Sulzman, J. \& Lamarque, J. F. Nitrogen deposition onto the United States and western Europe: synthesis of observations and models. Ecol. Appl. 15, 38-57 (2005).

26. Hansen, M. C. et al. High-resolution global maps of 21st-century forest cover change. Science 342, 850-853 (2013).

27. Mueller, T. et al. Human land-use practices lead to global long-term increases in photosynthetic capacity. Remote Sens. 6, 5717-5731 (2014).

28. Lehner, B. \& Döll, P. Development and validation of a global database of lakes, reservoirs and wetlands. J. Hydrol. 296, 1-22 (2004).

29. van der Werf, G. R. et al. Global fire emissions and the contribution of deforestation, savanna, forest, agricultural, and peat fires (1997-2009). Atmos. Chem. Phys. 10, 11707-11735 (2010).

\section{Acknowledgements}

This study was supported by the Strategic Priority Research Program (B) of the Chinese Academy of Sciences (Grant XDB03030404), National Basic Research Program of China (Grant 2013CB956303), National Natural Science Foundation of China (Grant 41530528), the 111 Project (Grant B14001), and the European Research Council Synergy grant ERC-SyG-610028 IMBALANCE-P. We thank all people and institutions who provided data used in this study, in particular, the TRENDY modelling group. R.B.M. is funded by NASA Earth Science. J.G.C. is grateful for support from the Australian Climate Change Science Program. A.A. and T.A.M.P. acknowledge support through EC FP7 grants LUC4C (Grant 603542) and EMBRACE (Grant 282672) and the Helmholtz Association ATMO programme, Y.W. acknowledges CSIRO strategic funding for CABLE science, E.K. was funded by ERTDF (S10) from the Ministry of Environment, Japan. J.M. is supported by the US Department of Energy (DOE), Office of Science, Biological and Environmental Research. Oak Ridge National Laboratory is managed by UT-BATTELLE for DOE under contract DE-AC05-00OR22725. B.D.S. is supported by the Swiss National Science Foundation and FP7 funding through project EMBRACE (282672).

\section{Author contributions}

S.Piao, R.B.M. and Z.Zhu designed the study. Z.Zhu performed the analysis. Z.Zhu, S.Piao, J.G.C., P.C. and R.B.M. drafted the paper. Z.Zhu, M.H., Z.Zeng, C.C., Y.Liu, H.Y. X.W., X.L., Y.P., Y.Li, R.L. and Z.X. collected data and prepared figures. S.S., P.F., A.A., B.D.S., B.P., C.K., E.K., J.M., J.P., L.C., N.V., N.Z., S.Peng, S.Z., T.A.M.P., and Y.W. ran the model simulations. All authors contributed to the interpretation of the results and to the text.

\section{Additional information}

Supplementary information is available in the online version of the paper. Reprints and permissions information is available online at www.nature.com/reprints.

Correspondence and requests for materials should be addressed to S.Piao.

\section{Competing financial interests}

The authors declare no competing financial interests. 


\section{Methods}

The growing season integrated leaf area index was used as a proxy of vegetation growth in this study. We identified the growing season for each $0.5^{\circ} \times 0.5^{\circ}$ grid cell of global vegetated area using GIMMS LAI3g data sets and freeze/thaw data sets. The growing season was first determined from the GIMMS LAI3g data set ${ }^{30}$ using a Savitzky-Golay filter and then refined by excluding the ground-freeze period identified by the Freeze/Thaw Earth System Data Record ${ }^{31}$. In particular, the growing season of evergreen broadleaf forests was set to 12 months and starts in January. All the satellite-observed leaf area products and leaf area index outputs of ecosystem models were first aggregated to $0.5^{\circ} \times 0.5^{\circ}$ spatial resolution and then composited to annual growing season integrated leaf area index data.

Three satellite-observed leaf area index products (GIMMS LAI, GLOBMAP LAI and GLASS LAI) were used to analyse the changes in global vegetation for the period 1982-2009. We used a nonparametric trend test technique (Mann-Kendall test) to evaluate trends in growing season integrated leaf area index derived from the three satellite LAI products at the $95 \%$ significance level. We analysed trends in LAI at pixel level, global level and continental level. When we tested trends in LAI at global and continental scales, we calculated the mean of LAI values of all the pixels in the specific region, weighting by the area of each pixel.

Ten ecosystem models were used to analyse the relative contributions of external driving factors to trends in global vegetation growth during 1982-2009. We performed four experimental simulations to evaluate the relative contribution of four main driving factors, namely, $\mathrm{CO}_{2}$ fertilization, climate change, nitrogen deposition and land cover change, to the global vegetation trends: (S1) varying $\mathrm{CO}_{2}$ only, (S2) varying $\mathrm{CO}_{2}$ and climate, (S3) varying $\mathrm{CO}_{2}$, climate and nitrogen deposition and ( $\mathrm{S} 4$ ) varying $\mathrm{CO}_{2}$, climate and land cover change. $\mathrm{S} 1, \mathrm{~S} 2-\mathrm{S} 1$, $\mathrm{S} 3-\mathrm{S} 2$ and $\mathrm{S} 4-\mathrm{S} 2$ were used to evaluate the effects of $\mathrm{CO}_{2}$ fertilization, climate change, nitrogen deposition and land cover change to vegetation growth, respectively (see Supplementary Information Section 7).

We used an optimal fingerprint method ${ }^{13}$ to detect the signals of $\mathrm{CO}_{2}$ fertilization, climate change, nitrogen deposition and land cover change effects simulated by ecosystem models at global scales. The optimal fingerprint expresses the observation $(\mathrm{Y})$ as a linear combination of scaled $\left(\beta_{i}\right)$ responses to external driving factors $\left(x_{i}\right)$, and internal variability $(\varepsilon): \mathrm{Y}=\sum_{i=1}^{n} \beta_{i} x_{i}+\varepsilon$. The scaling factors $\left(\beta_{i}\right)$ are estimated on the basis of the total least square method to adjust the amplitude of the responses of LAI to each driving factor. We regressed the satellite-observed LAI against responses of vegetation growth (expressed as LAI) to elevated atmospheric $\mathrm{CO}_{2}$, climate change, nitrogen deposition and land cover change estimated by multi-model ensemble mean simulations of ten ecosystem models. We also performed similar analysis for the simulated LAI under scenarios S1, S2, S3 and S4. These regressions provide best-estimate linear combinations of signals simulated by ecosystem models. The coefficients of the signals are the scaling factors $\left(\beta_{i}\right)$. A residual consistency test was introduced to check the consistency between the residuals of satellite-observed LAI and best-estimate combinations of signals and the assumed internal LAI variability ${ }^{13}$. The overall statistical model was considered suitable only if the residual consistency test passed at the $95 \%$ significance level. If the $95 \%$ confidence interval of the estimated scaling factor lies above zero, the signal of the corresponding driving factor is detected; the model simulations are suitable for attribution if the $95 \%$ confidence interval contains 1 .

\section{References}

30. Zhu, Z. C. et al. Global data sets of vegetation leaf area index (LAI) $3 g$ and fraction of photosynthetically active radiation (FPAR) 3g derived from global inventory modeling and mapping studies (GIMMS) normalized difference vegetation index (NDVI3g) for the period 1981 to 2011. Remote Sens. 5, 927-948 (2013)

31. Kim, Y., Kimball, J. S., McDonald, K. C. \& Glassy, J. Developing a global data record of daily landscape freeze/thaw status using satellite passive microwave remote sensing. IEEE Trans. Geosci. Remote Sensing 49, 949-960 (2011). 